\title{
AGRARREFORM UND GENOSSENSCHAFTSWESEN IN EINEM ENTWICKLUNGSLAND: ÄGYPTEN
}

\author{
Von BAsSAM TiBI
}

Insofern die sozio-ökonomisch schwach entwickelten Länder der Dritten Welt Agrargebiete mit überwiegender Landbevölkerung (60-80 Prozent der gesamten Population) sind, bildet die Agrarfrage für sie ein zentrales Problem auf allen Ebenen, der politischen, sozio-ökonomischen, demographischen u. a. Die Agrarfrage kann daher nur im Rahmen einer umfassenden Analyse der sozialen Verhältnisse des jeweils zu behandelnden Gebietes untersucht werden. Erst auf dieser analytischen Ebene können gesellschaftlich erforderliche Agrarreformen und die in sie eingebettete Bildung von Agrargenossenschaften sachgerecht diskutiert werden.

\section{Agrarreform und Agrargenossenschaft: Versuch einer Begriffsbestimmung}

Der Begriff Agrarreform wird in der wissenschaftlichen Diskussion sowohl als Bezeichnung für den Tatbestand benutzt, daß eine Landwirtschaft durch Anwendung neuer Methoden und durch Technisierung modernisiert wird, als auch dafür, daß eine Redistribution des Eigentums an Boden erfolgt. Indes erweisen sich beide begrifflichen Abgrenzungen als jeweils zu rigide. Eine Landwirtschaft mit starren feudalen oder semi-feudalen bzw. rückständigen agrarkapitalistischen Produktionsverhältnissen verweigert sich strukturbedingt einer umfassenden Modernisierung, die eine gesellschaftliche Umgestaltung und hohe Investitionen voraussetzt. So ist andererseits eine reine Redistribution, eine Parzellierung des Eigentums an Boden, die oft als Agrarreform ausgegeben wird, gewiß nicht der einzige erforderliche Weg, um ein Land aus seiner Rückständigkeit zu befreien.

Beide Fragen: die der Modernisierung der Landwirtschaft, um ihre Erträge zu maximieren, sowie die Eigentumsfrage, sind voneinander nicht $\mathrm{zu}$ trennen. Wir werden in der folgenden Diskussion über das Genossenschaftswesen sowie in unserer Fallstudie über Ägypten zeigen, daß umfassende Agrarinnovationen in Gebieten mit ausgeprägten Eigentums- und Herrschaftsverhältnissen nicht erfolgreich durchgeführt werden können. Eine Modernisierung der Landwirtschaft kann nicht ohne tiefgreifende Einschnitte in die Eigentumsstruktur erfolgen. Diese Eingriffe bleiben aber erfolglos, wenn sie nicht von vornherein an einer Modernisierung der Landwirtschaft orientiert sind. Parzellierung des Bodens heißt für sich genommen Sozialisierung der Armut und der Rückständigkeit ${ }^{1}$.

Theodor Bergmann zeigt in einer instruktiven Studie, daß in den meisten Entwicklungsländern mit feudalen und semi-feudalen Bodeneigentumsverhältnissen die ökonomisch herrschenden sozialen Schichten zugleich die staatlichen Herrschaftsinstanzen besetzt halten². Ein Staat mit einer solchen politischen Herr-

1 Ahnlich auch F. Kuhnen, „Die Bedeutung der Agrarreform im Rahmen der wirtschaftlichen Entwick-

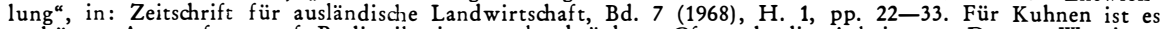
verkürzt, Agrarreform auf Redistribution zu beschränken. Cf. auch die Arbeit von Doreen Warriner, Land Reform in Principle and Practice, Clarendon Press, Oxford-London 1969. Von Warriner liegt auch eine beachtete Standardmonographie über Agrarreform in den arabischen Ländern vor: cf. Anm. 31). Zum eine beachtete Standardmonographie über Agrarreform in den arabischen Ländern vor: cf. Anm. 31). Zum
Stand der Diskussion über Agrarreform und Genossenschaftswesen cf. die Fachzeitschriften der Disziplin: Zeitschrift für das gesamte Genossenschaftswesen sowie Zeitschrift für ausländische Landwirtschaft, die laufend theoretische Beiträge, Fallstudien und Rezensionen über unsere Thematik veröffentlichen.

2 Theodor Bergmann, Funktionen und Wirkungsgrenzen von Produktionsgenossenschaften in Entwicklungsländern, Europäische Verlagsanstalt, Frankfurt/M. 1967, p. 46. 
schaftsstruktur wird freilich keine Gesetze erlassen, die Eingriffe in die Eigentumsverhältnisse beinhalten. Eine Änderung der Eigentumsverhältnisse erfordert also eine Anderung in der Herrschaftsstruktur, wie wir in dem materialen Teil über Ägypten illustrieren werden.

Wir wollen zunächst zwischen Boden- und Agrarreform differenzieren: Gelingt es, die politische Herrschaft der ökonomisch herrschenden Landaristokratie zu brechen, so ist die Möglichkeit gegeben, Gesetze zu erlassen, durch die das Eigentum an Boden limitiert und die Landaristokratie enteignet werden kann. Diese Gesetzgebung bezeichnen wir als Bodenreform. Unter Agrarreform verstehen wir im Anschluß an Wörz und Frauendorfer „sämtliche agrarpolitischen und agrarrechtlichen Maßnahmen ...., (die) auch die Umgestaltung der privaten Grundeigentumsverhältnisse einschließen, also die Bodenreform. Folglich ist der Begriff Bodenreform als eines der Mittel der Agrarreform zu definieren. Eine Agrarreform kann somit eine Bodenreform einschließen oder nicht, sie ist immer dann gegeben, wenn durch mehrere Maßnahmen wesentliche Züge der gesamten Agrarverfassung geändert werden" 3 .

Die Diskussion über das Agrargenossenschaftswesen erscheint, im Gegensatz zur relativ unumstrittenen obigen Deutung des Agrarreformbegriffes, weit komplexer angesichts der Fülle divergierender Lehrmeinungen innerhalb der genossenschaftstheoretischen Literatur. Wir wollen uns hier nicht mit Formaldefinitionen aufhalten, zumal sie - das zeigt die Fachdiskussion unter den Genossenschaftstheoretikern - kaum fruchtbar sind. Ubberblicke über Versuche, die Agrargenossenschaften mit Formalbezeichnungen in den Griff zu bekommen, liegen außerdem bereits vor, so daß wir uns hier nicht zur Bestandsaufnahme veranlaßt sehen ${ }^{4}$. Vielmehr wird im folgenden, gestützt auf die Beiträge von zwei herausragenden Genossenschaftstheoretikern: Trappe und Bergmann, eine inhaltliche Diskussion darüber geführt, was unter Genossenschaft zu verstehen sei, um sodann den Zusammenhang von Agrargenossenschaften und Agrarreform zu vergegenwärtigen.

Die Genossenschaftstheoretiker Bergmann ${ }^{5}$ und Trappe $^{6}$ gelangen nach eingehender Sichtung der theoretischen Diskussionen über Agrargenossenschaften zu dem Resümee, daß über das Thema angesichts der einander widersprechenden Lehrmeinungen nur Konfusion herrscht. Bergmann konstatiert, daß die Genossenschaftsformen variieren und zudem sehr wandelbar sind. Wage man eine Definition, so müsse sie „sehr allgemein sein, wenn sie alle Erscheinungsformen zu erfassen

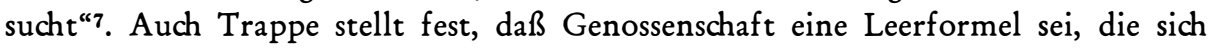
„auf der Basis relevanter Tatsachen und ideologischer Faktoren auf sehr unterschiedliche Weisen, aufladen" " lasse ${ }^{8}$. Klar sei der Genossenschaftsbegriff nur in Rechtsordnungen, wo er sich auf Legaldefinitionen stützt. An anderer Stelle zeigt Trappe, daß jeweils disziplin-orientierte Fassungen des Begriffs bestehen ${ }^{9}$, und Bergmann verweist darauf, daß es selbst innerhalb der einen Disziplin Agrarökonomie eine Fülle von einander widersprechenden Begriffsbestimmungen gibt ${ }^{10}$.

3 Johannes G. F. Wörz, Die genossenschaftliche Produktionsförderung in Ägypten, Ernst Klett Verlag, Stuttgart 1967, p. 31.

4 Th. Bergmann, Funktionen . . . , op. cit., pp. 9-18.

5 Bergmann, op. cit.

6 Paul Trappe, „Zur Entwicklungsfunktion des Genossenschaftswesens unter Berücksichtigung vorgegebener Sozialstrukturen ${ }^{\text {, }}$ in: Zeitschrift für die gesamte Staatswissenschaft, Bd. 118 (1962), H. 3, pp. 442-461.

7 Bergmann (Anm. 2), p. 9.

8 Trappe (Anm. 6), p. 447.

9 Paul Trappe, Die Entwicklungsfunktion des Genossenschaftswesens am Beispiel ostafrikanischer Stämme, Luchterhand Verlag, Berlin-Neuwied 1966, pp. $146 \mathrm{f}$.

10 Bergmann, Funktionen... op. cit. 
Dieser Tatbestand sollte jedoch keinen Anlaß geben, den Versuch einer allgemeinen, jedoch inhaltlichen Bestimmung der Genossenschaft, die sich auch in juristischen Formen niederschlagen kann, zu unterlassen. Wir gehen im folgenden davon aus, daß der Genossenschaftsbegriff, insofern er das körperschaftliche Moment berührt, ein juristischer Begriff ist, folgen aber Trappe, wenn er feststellt, „daß der Begriff der Genossenschaft eo ipso eine soziologische Kategorie ist. Genossenschaft deckt nicht nur einen besonderen Sachverhalt der sozialen Wirklichkeit, sondern Genossenschaft entspricht semantisch einer bestimmten sozialen Struktur"11. Mit Trappe betonen wir, daß der Vorwurf des Soziologismus, würde er erhoben, hier an falscher Stelle wäre.

Die Struktur einer Genossenschaft ist egalitär; denn Genossenschaft ist ein „soziales Gebilde mit den wesentlichen Begriffsmerkmalen der Gruppe von Gleichberechtigten und des festen, d. h. an eine bestimmte Aktion gebundenen und relativ dauerhaften Zusammenschlusses"12. Genossenschaftliche Strukturen stehen folglich im Gegensatz zu hierarchischen, seien sie feudal oder kapitalistisch; „mit dem Genossenschaftlichen wird gemeinhin ein demokratisches, mit dem herrschaftlichen ein hierarchisch geschichtetes Sozialmodell verbunden"13. In einem diesen Ausführungen vorausgehenden Aufsatz hat Trappe den vorläufigen Versuch unternommen, genossenschaftliche Momente in bereits existierenden Sozialstrukturen in Afrika aufzuspüren; er kam zu dem Ergebnis, daß die segmentären Gesellschaften der "Naturvölker" keine zentrale Herrschaftsinstanz kennen, daß bei ihnen also nicht von legaler Herrschaft im Sinne Max Webers gesprochen werden könne ${ }^{\mathbf{1 4}}$. Gerade diese egalitäre Konstitution der Sozialstrukturen von segmentären Gesellschaften läßt nach Trappe das Attribut "genossenschaftlich“ für sie zu15. Trappes Forschungen kreisen seither um die Möglichkeiten der Entwicklung von in vorgegebenen Sozialstrukturen vorhandenen genossenschaftlichen Momenten. Damit entlastet er sich allerdings von der Untersuchung der Möglichkeiten einer genossenschaftlichen Entwicklung in Gesellschaften mit zentraler Herrschaftsinstanz. Da sein Ansatz nur für herrschaftsfreie bzw. herrschaftsschwache Gesellschaften gilt, weiß Trappe auch keine Antwort auf außerhalb dieses Bereiches bestehende Fragen. Er begnügt sich mit der zwar berechtigten Feststellung, daß eine genossenschaftliche Entwicklung nur durch Partizipation und Mobilisierung breiter Bevölkerungsmassen zu realisieren ist und daß in Ländern, „wo sich ein derartig entwicklungsfeindliches Schichtungsmodell in einer ihm adäquaten entwicklungsfeindlichen Rechtsordnung spiegelt", die Voraussetzungen hierzu fehlen; einen Ausweg für diese Länder kann Trappe aber aufgrund seines verkürzten Ansatzes nicht empfehlen ${ }^{\mathbf{1 6}}$.

Anders als Trappe geht Bergmann ${ }^{17}$ detailliert auf Probleme des Genossenschaftswesens in Gesellschaften mit zentraler Herrschaftsinstanz ein und diskutiert die

11 Trappe, Die Entwicklungsfunktion . . . (Anm. 9), op. cit., p. 147.

12 Ibid., p. 148.

13 Ibid., p. 149.

14 Cf. Trappe (Anm. 6), pp. 448 ff. Es ist das Verdienst von Ch. Sigrist, eine gründliche Studie über diese segmentären Gesellschaften vorgelegt zu haben; cf. Ch. Sigrist, Regulierte Anarchie, Untersuchungen zum Fehlen und zur Entstehung politischer Herrschaft in den segmentären Gesellschaften Afrikas. Walter Verlag, Olten-Freiburg 1967; cf. auch die kritische Würdigung von M. O. Hinz, „Die Ordnung der regulierten Anarchie“, in: Kölner Zeitschrift für Soziologie und Sozialpsychologie, Bd. 23 (1971), H. 1, pp. $110-117$.

15 Trappe (Anm. 6), p. 451.

16 So etwa Trappes Habilitationsschrift (Anm. 9); cf. hierzu kritisch B. Tibi, „Kolonialherrschaft, Antikolonialismus und Dekolonisation. Das afrikanische Exempel“, in: Neue Politische Literatur, Bd. 15 (1970), H. 4, pp. 507-532, hierzu pp. 529 f.

17 Bergmann (Anm. 2). Um den Anmerkungsapparat nicht zu überlasten, nennen wir entsprechende Seitenzahlen aus Büchern, die im Text detailliert behandelt werden, direkt hinter den Zitaten und weisen darauf in der Fußnote bei Erwähnung der Quelle hin. Die Seitenzahlen hinter den folgenden Zitaten beziehen sich alle auf die zitierte Quelle von Bergmann (Anm. 2). 
gegebenen Schwierigkeiten, ausgehend von seiner eigenen Bestimmung der Agrargenossenschaften.

Bergmann vertritt eine historisch orientierte Position; er betont, daß kein allgemeines Leitbild für den Aufbau von Agrargenossenschaften zu formulieren sei. Vielmehr solle für jedes Land ein seinen eigenen Verhältnissen entsprechendes Genossenschaftsmodell entwickelt werden (p. 7). Diese Einschränkung hindert Bergmann jedoch nicht daran, eine allgemeine Aussage über Genossenschaften in Entwicklungsgesellschaften zu machen. Er differenziert generell zwischen Vollgenossenschaften, i. e. solche, bei denen keine individuelle Mitgliedwirtschaft betrieben wird; sie seien für die Lösung von Entwicklungsaufgaben zweckdienlicher als hilfswirtschaftliche Genossenschaften, i. e. solche, bei denen der einzelne Mitgliedsbetrieb ökonomisch gewichtiger und die Genossenschaft nur Hilfsbetrieb ist (pp. 16, 85). Die Form der Vollgenossenschaft sorge eher „für die schnelle Verbreitung neuer Produktionsmethoden, für den Einsatz teurer und knapper Produktionsmittel“" usw. (p. 94).

Unter Genossenschaft versteht Bergmann „die gleichberechtigte, verbindlich institutionalisierte Zusammenarbeit in verbundwirtschaftlichen Organisationen“ (p. 17). Wie Trappe hebt Bergmann die egalitäre Dimension der Genossenschaften hervor. Diese Dimension „grenzt die Genossenschaften gegen andere Formen der wirtschaftlichen Zusammenarbeit ab, bei denen der Einfluß auf Organisation meistens vom eingebrachten Kapital abhängt. Dadurch wird auch die soziale Funktion der Genossenschaft deutlich" (p 18). Nicht nur, weil die Agrarproduktion, strukturell bedingt, die Konzentration in große Einheiten wie in der Industrie verbietet (cf. p. 23), sondern auch wegen der Wahrung der Freiheitssphären der Mitglieder einer Genossenschaft ist Bergmann gegen Kollektivierungsversuche in der Landwirtschaft, wie sie in der Sowjetunion praktiziert wurden. Das Prinzip des freiwilligen Beitritts muß Bergmann zufolge konstitutiv bleiben. Doch ist Bergmann nicht so naiv, Systemgrenzen, die diesem Prinzip im Weg stehen, zu übersehen. Es gibt zwei Möglichkeiten, dieses Prinzip in Entwicklungsgesellschaften zu unterlaufen, die auch durch entsprechende Rechtsordnung zementiert werden: „Es kann Druck oder Zwang von der Genossenschaft in Richtung auf Beitritt ausgeübt werden, aber auch von einem Grundbesitzer gegen seinen Pächter, von einem Geldverleiher oder Händler gegen seinen Schuldner, um den Beitritt zu einer Genossenschaft zu verhindern (p. 32).“

In vielen Entwicklungsländern mit ausgeprägter feudaler Agrarstruktur ist es um die Freiwilligkeit des Bauern schlecht bestellt. Denn wie soll eine Genossenschaft aufgebaut werden, wenn folgende Verhältnisse herrschen: „Die Masse der Dorfbewohner in diesen Ländern ist unterbeschäftigt, aber auch unterernährt. Die Bereitstellung der für eine langfristige Arbeitsinvestition notwendigen Lebensmittel übersteigt die Produktionskraft des einzelnen Landwirtschafters. Die bewirtschaftete Fläche ist $\mathrm{zu}$ klein und $\mathrm{zu}$ stark parzelliert, die überschüssige Arbeitskraft der Einzelfamilie zu gering, um größere Verbesserungen durchzuführen und zu nutzen ... (p. 33). „Die Landbewirtschafter verfügen rechtlich nicht über den Boden, dürfen diesen also nicht in eine Vollgenossenschaft einbringen. Sie haben zu wenig Kapital, um das Startkapital einer Dienstleistungsgenossenschaft einzuzahlen. Sie sind so verschuldet, daß sie nicht vom privaten Kommissionär und Geldverleiher loskommen können. Ein eigener freier Entschluß zur Bildung einer Genossenschaft ist daher oft nicht möglich (pp. 34 f.)." Staatliche Eingriffe in eine solche Situation können nur zugunsten der Großgrundbesitzer ausfallen, zumal in einer 
Reihe von Entwicklungsländern „die Beamtenschaft aus der Grundbesitzer- oder Steuerpächterschicht (kommt). Für viele von diesen bedeutet es eine unzumutbare Selbstverleugnung, eine Landreform durchzuführen und mitzuhelfen, ländliche Genossenschaften zu bilden (p. 46)“. Wird in einem solchen Staat (z. B. im Iran) eine Agrarreform durchgeführt, dann geschieht das nur, um eine soziale Revolution zu verhindern, und es wird in der Regel an der Struktur kaum etwas geändert.

Bergmann tritt dafür ein, daß der Staat Maßnahmen zur Bildung von Genossenschaften ergreift. Denn „hält der Staat sich fern, können Genossenschaften kaum entstehen. Hilft er mit, gewinnt er automatisch einen beträchtlichen Einfluß, der die spätere Genossenschaftsdemokratie präjudizieren kann (p. 43)“. Angesichts dieses Dilemmas verlangt Bergmann die Beibehaltung des genossenschaftlichen Prinzips der Freiwilligkeit und der Eigeninitiative. Denn ansonsten werde die Genossenschaft „zu einem verlängerten Arm der Staatsverwaltung, der weniger die Mitglieder als die staatlichen Interessen fördert. Von da bis zum administrativen Zwang ist der Schritt nicht allzuweit, sofern ein Staatsapparat vorhanden ist, der über geeignete Mittel verfügt (p. 43)“. Nur in segmentären Gesellschaften, die keine zentrale Herrschaftsinstanz kennen, bleibt dies völlig erspart ${ }^{18}$ (cf. p. 41; auch Anm. 14 dieser Arbeit).

Neben der subsidiären Rolle des Staates, die nicht die Selbstverwaltung und -bestimmung der Genossenschaften einschränken soll, ist für das Funktionieren der Genossenschaften die Befolgung zweier Kriterien bei der Aufnahme der Mitglieder notwendig: „Eine regionale oder örtlich festzulegende oberste Besitzgrenze und körperliche Mitarbeit (p. 37)." Hierzu gehört, daß aus den Eigentumsrechten an dem eingebrachten Kapital keine Sonderrechte abgeleitet werden dürfen. Denn ansonsten sind zwar „die aus dem Eigentum hergeleiteten Rechte . . voll geschützt, eines der wesentlichen Genossenschaftsprinzipien aber aufzugeben. Außerdem besteht dann die Gefahr, daß Genossen gerade in den arbeitsreichen Zeiten private Lohnarbeit suchen und deshalb der Genossenschaft fernbleiben (p. 40)“. Neben diesen Einschränkungen führt Bergmann auch die restriktive Verteilung von Bodenreformländereien an. Zwar soll die Mitgliedschaft in den Genossenschaften frei sein, dies soll aber nicht für Bodenreformland gelten. „Die Zuteilung von Bodenreformland ist jedoch an die Mitgliedschaft gebunden; die Freiwilligkeit ist also bedingt (p. 35)." Würde man nicht nach dieser Maxime verfahren, dann fände man sich einem Zustand ausgesetzt, in dem der Boden parzelliert und die Produktivität beeinträchtigt ist.

\section{Agrarreform und Genossenschaftswesen in Ägypten}

Um den historischen Stellenwert der ägyptischen Agrarreformgesetzgebung und der mit ihr verbundenen Agrarneuordnung zu demonstrieren, skizzieren wir zunächst die zentralen Aspekte der Agrarfrage in Ägypten. Nach einer kurzen Darstellung des Agrarreformgesetzes vom September 1952 diskutieren wir sodann die Wirkung dieses Gesetzes sowie dessen Erweiterung durch die zweite Agrarreform von 1961.

18 In diesem Zusammenhang erscheint das Experiment der chinesischen Volkskommunen interessant, obwohl China keine segmentären Gesellschaften aufweist. Cf. hierzu Max Biehl, Die chinesische Volksrepublik im "Großen Sprung“ und danach. Verlag Weltarchiv, Hamburg 1965, und dazu die kritische Würdigung von K. Steinhaus, „Deutschsprachige Literatur über die Volksrepublik China“, in: Das Argument, Bd. 9 K. Steinhaus, „Deutschsprachige Literatur über die Volksrepublik China“, in: Das Argument, Bd. 9
(1967) H. 43, pp. 166-181, hierzu pp. $174 \mathrm{ff}$. Ebenso interessant ist das algerische Experiment, wo die (1967) H. 43, pp. 166-181, hierzu pp. $174 \mathrm{ff}$. Ebenso interessant ist das algerische Experiment, wo die
Bauern nach dem Prinzip der Selbstverwaltung (Autogestion) ohne staatliche Bevormundung die Genossenschaften betreiben konnten, bis die Armee und die Bürokratie dies unterhöhlten; cf. W. Plum, Der Niedergang der Agrarreform in Algerien ${ }^{\star}$, in: Geist und Tat, Bd. 22 (1967), pp. 94-99; ders., Sozialer Wandel im Maghreb, Verlag für Literatur und Zeitgeschehen, Hannover 1967, pp. $133 \mathrm{ff}$. 
Aufgrund der hydraulischen Struktur seines Agrarsystems war Ägypten seit der Pharaonenzeit ein zentral regiertes Land, zumal die hydraulischen Aufgaben nur zentral und nicht auf lokaler Ebene zu bewältigen waren. Das dieser gesellschaftlichen Struktur entsprechende Bodenrecht kennt nur staatliches Eigentum. Auch als im 7. Jahrhundert n. Chr. die Araber Agypten eroberten, blieb diese Ordnung unverändert. Dies gilt ebenso für die osmanische Eroberung 151720. Abdel-Malek zeigt einige Nuancen auf, die dieses System im Verlauf der Geschichte erfuhr. So gab es sowohl in der Pharaonen- als auch in der Araber-Zeit in Agypten ein Nutznießersystem, wonach. Instrumente der Zentralinstanz - wie die Priesterschaft und Militärführer - mit Boden belehnt wurden. Die Mamluken waren diejenigen Militärführer in der ägyptischen Geschichte, die sich aufgrund des ihnen überlassenen Bodens zeitweise verselbständigten. Die Zentralinstanz vermochte jedoch ihre Autorität zu restaurieren. Außer in diesen Fällen der Belehnung nach dem Nutznießersystem gab es keinen Grundbesitz ${ }^{21}$. Unter osmanischer Herrschaft gedieh in Ägypten das Iltizam-System (Lehns-, Pachtsystem). Das in ihm herrschende Bodenrecht sah Ämter zur Steuereintreibung vor; diese Ämter konnten gegen Zahlung bestimmter Summen auf begrenzte Zeit von der Zentralbehörde auf Personen übertragen werden. In der Praxis wurden die Ämter jedoch vererbt und somit auch der in ihrem Geltungsbereich liegende Boden. Von privatem Eigentum an Boden kann indes noch keine Rede sein, da die Zentralbehörde als rechtliche Alleineigentümerin des Bodens den Multazimin jederzeit ihr IltizamPrivileg entziehen konnte ${ }^{22}$. Dieser spezifische „orientalische“ Feudalismus unterscheidet sich also wesentlich vom Feudalsystem europäischen Typs ${ }^{23}$.

Unter der Herrschaft Muhammad 'Alis, unter der Ägypten sich faktisch vom Osmanischen Reich loslöste, wurde das Iltizam-System durch Staatsgewalt abgeschafft und das Eigentum an Boden wurde. wieder völlig zum Staatsmonopol24. Der ägyptische Sozialhistoriker Ibrahim 'Amer bezeichnet das System der agrarischen Ausbeutung zur Zeit Muhammad 'Alis als „ein temporäres System des Úbergangs zwischen Feudalismus und Kapitalismus“"25.

Mit Beginn des kolonialen Einflusses in Agypten entstand dort auch ein modernes Bodenrecht, das Eigentum an Boden kennt ${ }^{26}$. Schon 1840 zwangen die Kolonialmächte Ägypten, das Staatsmonopol aufzulösen, und 1846 wurde ein Gesetz erlassen, das 1. eine begrenzte Form des Eigentums zuließ, 2. eine Belastung durch Hypotheken erlaubte und 3. die Úbertragung des Nutzungsrechtes an eine dritte Partei gestattete.

19 Cf. zum folgenden Annouar Abdel-Malek, Agypten: Militärgesellschaft. Das Armeeregime, die Linke und der soziale Wandel unter Nasser, Suhrkamp Verlag, Frankfurt/M. 1971, pp. 94 ff., sowie Saad Khalil, Grundeigentum und Landreform in Ägypten. Diss. rer. pol., Bonn 1959, pp. $20 \mathrm{ff}$.

20 Abdel-Malek (Anm. 19), p. 96; Khalil (Anm. 19), p. 21. Zur Hydraulik-Problematik cf. das Werk von Karl A. Wittogel, Die orientalische Despotie, Kiepenheuer \& Witsch, Köln-Berlin 1962.

21 Abdel-Malek, op. cit., pp. $96 \mathrm{f}$

22 Ibid., p. 99 .

23 Cf. ibid.

24 Insgesamt zur Agrarpolitik des Muhammad-'Ali-Regimes cf. die umfangreiche Monographie von Helen Anne B. Rivlin, The Agricultural Policy of Muhammad Ali of Egypt, Harvard Uni. Press, Cambridge/ Mass. 1966. Cf. auch B. Tibi, Nationalismus in der Dritten Welt am arabischen Beispiel, Europäische Verlagsanstalt, Frankfurt/M. 1971, pp. $64 \mathrm{ff}$.

25 Ibrahim 'Amer, al-Ard wa'l-fallah. al-Mas'ala az-zira'iyya fi Misr (Der Boden und der Bauer. Die Agrarfrage in Agypten), Kairo 1958, hier zit. nach Abdel-Malek (Anm. 19), pp. 100 f. Leider blieb uns diese wichtige Quelle, auf der Abdel-Maleks Analyse zum guten Teil $f u ß t$, im Original unzugänglich.

26 Die beste Quelle hierüber ist bisher Gabriel Baer, A History of Landownership in Modern Egypt 1800-1950. Oxford Uni. Press, London 1962; cf. auch B. Tibi (Anm. 24), pp. $64 \mathrm{ff}$. 
Dieses Gesetz wurde 1858 durch ein von Muhammad 'Alis Sohn erlassenes Gesetz erweitert, und schließlich erließ der Khedevi Isma'il (1863-1879) im Jahr 1871 ein Gesetz, wonach die Úbertragung von Grundeigentum gegen die Vorauszahlung einer. Sechsjahressteuer möglich. wurde. Aufgrund dieser. Einschränkung war der Erwerb von Eigentum an Boden der khedivalen Familie und der aus der. Staatsbürokratie hervorgegangenen Landaristokratie vorbehalten. Erst nach der Okkupation Ägyptens durch die britische Kolonialmacht (1882) wurde das volle Recht auf Grundeigentum auf die gesamte Bevölkerung - durch das Gesetz von 1891 übertragen. „Mit dieser Maßnahme gelang es ihr (der Kolonialmacht, B.T.) zugleich, die Bauern, i. e. die Mehrheit der Bevölkerung, an das. Land zu binden und. die Ersparnisse und existierenden Kapitalien auf den Landkauf zu lenken, ein Vorgang, der besonders durch die Drosselung der Industrialisierung angeregt wurde ${ }^{27}$."

Es wäre falsch, diese Entwicklung als eine Rückkehr zum Feudalismus zu bezeichnen. Vielmehr ist das seinerzeit geschaffene Grundeigentum in ein System des agrarischen Kapitalismus einzuordnen. Der Boden wurde zum bevorzugten Marktartikel, und die Ersparnisse der Stadtbevölkerung dienten immer mehr dem .Landkauf. Es entstand eine spezifische Besitzstruktur. So verfügten 1894 etwa 84 Prozent der Grundbesitzer über 21 Prozent des Landes (ihr Besitz betrug. jeweils unter 5 Feddan; 1 Feddan $=0,4201$ ha). Dagegen verfügten etwa 1,5 Prozent der Grundbesitzer über 44 Prozent des Landes (wobei der Besitz je über 5.0 Feddan betrug); aus dieser Schicht der Großgrundbesitzer ragte eine Landaristokratie deutlich hervor: 280 Landbesitzer, i. e. 0,01 Prozent der Grundbesitzer hatten 10. Prozent des Landes inne28. Bis 1952 fand innerhalb des Großgrundbesitzes ein Konzentrationsprozeß statt. Die Konzentration des Großgrundbesitzes führte aber nicht zur Entstehung von Großplantagen, da die Großgrundbesitzer ihre Ländereien nicht selbst verwalteten: Sie verpachteten ihren Besitz auf Versteigerungen. Die Pächter parzellierten das. Land und verpachteten es weiter an Bauern zu höheren Preisen als die, die sie selbst für die Pacht zahlen mußten, um sich auf diese Weise zu bereichern. So entstand ein System der Vermittler der Vermittler, in dessen Rahmen nicht nur eine große parasitäre Schicht neben der der Großgrundbesitzer: die Pächter, entstand, sondern auch der Boden vollends parzelliert wurde und die meisten Betriebseinheiten unwirtschaftlich arbeiteten ${ }^{29}$. Die Opfer dieses Systems waren die kleinen Bauern und Landarbeiter, wenn auch die gesamte Bevölkerung an der mit diesem parasitären System verbundenen ökonomischen Stagnation litt.

Bereits in den vierziger Jahren wurde in der Opposition und in Intellektuellenkreisen die Parole der Agrarreform laut ${ }^{30}$. Von dem royalistischen Regime war indes keine Agrarreformgesetzgebung zu erwarten, denn die Königsfamilie war der größte Großgrundbesitzer des. Landes.

b) Die Agrargesetzgebung vom 9. September 1952

Als die Gruppe der „Freien Offiziere“ durch ihren Coup d'état vom 23. Juli 1952 die Macht ergriffen und der Monarchie ein Ende gesetzt hatte, besaßen ihre Ange-

\footnotetext{
27 Khalil (Anm. 19), p. 24. Es ist in diesem Zusammenhang erwähnenswert, daß Muhammad 'Alis Politik der Industrialisierung Agyptens durch die Kolonialpolitik der Großmächte vereitelt wurde. Zur Industrialisierungspolitik Muhammad 'Alis cf. Zvi Y. Hershlag, Introduction to the Economic History of the Middle East, Brill Verlag, Leiden 1964, pp. $85 \mathrm{ff}$.

$28 \mathrm{Cf}$. hierzu Abdel-Malek (Anm. 19), pp. $101 \mathrm{ff}$; dort auch ein allgemeiner Uberblick über die Vierteilung des Grundbesitzes zwischen 1894 und 1952.

29 Detaillierte Argaben hierüber finden sid̀ bei El-Shagi El-Shagi, Neuordnung und Bodennutzung in Ägypten, Weltforum Verlag, München 1969, pp. 26-28, und bei Khalil (Anm. 19), pp. $24 \mathrm{ff}$., $30 \mathrm{ff}$., $34 \mathrm{ff}$. 30 Hierüber Abdel-Malek (Anm. 19), pp. $110 \mathrm{ff}$.
} 
hörigen, wie Abdel-Malek und viele andere Ägypten-Experten unterstreichen, "weder ein detailliertes Programm noch eine theoretische Konzeption des zukünftigen Ägyptens"31. Auch eine Agrarreform hatten sie nicht im Auge. Erst die Unruhe und die militanten Aktionen der Massen, die den Staatsstreich für eine Revolution hielten und große Hoffnungen an ihn knüpften, und erst die Konfrontation des Militärregimes mit der ihm opponierenden Landaristokratie waren die Ursache für den Erlaß des Gesetzes Nr. 178 vom 9. September 1952 über eine Agrarreform. Die Reformgesetzgebung war primär eine politische Entscheidung; sie war einmal eine Präventivmaßnahme, die der Massenbewegung Einhalt gebieten sollte, besonders nach dem Arbeiteraufstand von Kafr ad-Dauwar, dessen Führer die "Freien Offiziere" hinrichten ließen. Und auf ein weiteres politisches Problem verweist die Agrarreform von 1952, „nämlich das der Staatsmacht, die von der Landaristokratie ausgeübt wurde und deren Privilegien die Armee zugunsten des industriellen Flügels zu reduzieren beabsichtigte“s2.

Das ägyptische Gesetz Nr. 178 vom 9. Sept. 1952 über Agrarreform besteht aus sechs Abteilungen ${ }^{33}$ :

Teil I (Artikel 1-17) betrifft die Limitierung des Grundbesitzes und Redistribution des konfiszierten Grundbesitzes an die kleinen Bauern. Nach Art. 1 dürfen Privatpersonen nicht mehr als 200 Feddan Land besitzen. Art. 2 nimmt davon Körperschaften, insbesondere Industriewerke, Agrarunternehmen, die Odland fruchtbar machen; Stiftungen und wissenschaftliche Vereinigungen aus. Art. 3 bestimmt, daß der über der zugelassenen Grenze liegende Boden innerhalb von 5 Jahren von der Staatsgewalt eingezogen wird und daß es den Grundbesitzern überlassen ist zu entscheiden, welches Land sie behalten, welches sie abgeben. Art. 4 erlaubt den Großgrundbesitzern, zusätzlich zu den 200 Feddan Boden 50 Feddan pro Kind, höchstens jedoch 100 Feddan zu behalten. Art. 5 und 6 gehören zu den wichtigsten Bestimmungen; sie enthalten Regelungen über die Entschädigung der Großgrundbesitzer: Jeder Großgrundbesitzer erhält für das ihm genommene Land Staatsobligationen, die den zehnfachen Pachtwert seines Landes haben, neben einer Entschädigung für die auf dem Land liegenden Werte (Bäume und Bauten). Diese Obligationen werden mit 3 Prozent jährlich auf 30 Jahre Dauer verzinst. Mit diesen Staatsobligationen können die Großgrundbesitzer Steuern bezahlen und Odland erwerben. Die restlichen Artikel befassen sich mit Ausführungsbestimmungen und diktieren, daß das Land nur an solche Personen verteilt werden darf, die es selbst bebauen, und daß die zur Zeit des Gesetzeserlasses auf diesem Land wirkenden Bauern bevorzugt werden.

Teil II (Artikel 18-22) befaßt sich mit Agrargenossenschaften. Hierauf gehen wir in dem den Genossenschaften gewidmeten Abschnitt weiter unten ein.

31 Ibid., p. 93; cf. auch Doreen Warriner, Land Reform and Development in the Middle East, Oxford Uni. Press, London $1962^{2}$ (zuerst 1957; hier überarbeitet und erweitert), p. 10. Zur nasseristischen Ideologie cf. B. Tibi (ed.), Die arabische Linke, Europäische Verlagsanstalt Frankfurt/M. 1969, pp. 31 ff. sowie ders., "Armee und sozialer Wandel in Nordafrika", in: Afrika Spectrum, Bd. 6 (1971), H. 1, pp. $34-55$.

32 Abdel-Malek (Anm. 19), p. 116.

33 Der Text des Gesetzes einschließlich seiner Ergänzungen findet sich ungekürzt im Anhang zu Sayid Marei, al-Islah az-zira'i fi khams sanawat (Die Agrarreform nach fünf Jahren), Kairo 1957, pp. 79 ff, wonach wir hier zitieren. Marei war seinerzeit Agrarreform-Minister und mußte wegen einer Bestechungsaffäre zurücktreten, wurde aber 1962 rehabilitiert. Erläuterungen und eine Zusammenfassung des Gesetzes finden sich bei Khalil (Anm. 19), pp. 67 ff., Abdel-Malek (Anm. 19), pp. 117 ff., und Warriner (Anm. 31), pp. $31 \mathrm{ff}$. Cf. auch Hussein Mohamed Abdel Samie, Agrarreform und Maßnahmen zur Förderung der landwirtschaftlichen Entwicklung der VAR und des Irak, landwirtschaftliche Diss., Kiel 1960, pp. $42 \mathrm{ff}$; Saad M. Gadalla, Land Reform in Relation to Social Development. Egypt. University of Missouri Press, Columbia 1962, pp. 31 ff., sowie die umfassende Quelle von Gabriel S. Saab, The Egyptian Agrarian Reform 1952-1962, Oxford Uni. Press, London 1967, pp. $17 \mathrm{ff}$. 
Teil II (Artikel 23-24) bestimmt, daß der Boden nicht in Einheiten unter 5 Feddan aufgeteilt werden darf. Diese Vorschrift konnte allerdings kaum in die Praxis umgesetzt werden, weil die Erben meistens nicht bereit waren, auf ihren Anteil zu verzichten. Das Gesetz wurde auf verschiedene Weisen umgangen.

Teil IV (Artikel 25-30) befaßt sich mit den zusätzlichen Steuern.

Teil V (Artikel 31-37) regelt die Beziehungen zwischen Pächtern und Großgrundbesitzern.

Teil VI (Artikel 38-40) enthält Schutzklauseln für die Landarbeiter.

c) Vom Inkrafttreten des ersten Agrarreformgesetzes 1952 bis zur zweiten ägyptischen Agrarreform 1961

Sieht man von den Werken meist ägyptischer Autoren ab, die kritiklos die staatlichen ägyptischen Versionen über die erste ägyptische Agrarreform von 1952 übernehmen ${ }^{34}$, so besteht in der Literatur - trotz der verschiedenen politischen Wertorientierung der Autoren ${ }^{35}$ - Ubereinstimmung darüber, daß diese Agrarreform, bedingt durch ihren Charakter, mit Versagen gekrönt war.

Nach dem Gesetz von 1952 durften die Großgrundbesitzer praktisch 300 Feddan Land behalten. Und da das Gesetz ihnen auch die Möglichkeit gab, den Teil, den sie abzugeben hatten, selbst auszuwählen, haben die Großgrundbesitzer freilich meist das fruchtbare, mit Bäumen und Bauten ausgestattete Land behalten und die schlechten Teile abgegeben. Hinzu kommt, daß die Großgrundbesitzer es vermochten, durch Bestechungen mehr Land zu behalten, als ihnen nach dem Gesetz zustand, bzw. die Abgabe der Ländereien über 300 Feddan relativ lang hinauszuzögern ${ }^{36}$.

Zwei wichtige Faktoren haben die erste ägyptische Agrarreform neben den bereits genannten Gründen neutralisiert: Einmal wurde das konfiszierte Land zunächst Staatseigentum und wurde nicht an die Bauern verteilt. So wurden - nach staatlichen Angaben - von 5964000 Feddan fruchtbaren Bodens nur 645642 Feddan an 226000 , d. h. 10 Prozent der zwei Millionen Bauern verteilt. Der Staat machte 1955 aus dem Agrarreformboden, d. h. dem noch nicht verteilten konfiszierten Boden einen Profit von £E 2754 800, so daß Abdel-Malek sich veranlaßt sieht zu sagen, daß der ägyptische Staat „den Platz der reichen Grundbesitzer in der Ausbeutung der armen Bauern und Landarbeiter eingenommen hatte" 37 . Zum anderen war die Entschädigung so großzügig geregelt, daß die Großgrundbesitzer nach Berechnungen Warriners den Preis ihres Bodens in etwa 14 Jahren amortisieren konnten ${ }^{38}$. Die mit dieser Entschädigung verbundene Hoffnung des ägyptischen Militärregimes, daß die Investitionen sich vom Agrar- auf den Industriesektor verlagern würden, erwies sich als Illusion, denn die Verlagerung erfolgte auf den Bausektor: Die Großgrundbesitzer bauten Prachtvillen und Luxusappartements in einem solchen Ausmaß, daß die Investitionen auf dem Bausektor 1955 47,3 Prozent aller Investitionen und 75,8 Prozent aller Privatinvestitionen ausmachten. Dies veranlaßte die Militärregierung im Jahr 1956 dazu, das Gesetz Nr. 344 zu erlassen, mit dem die Investitionen auf dem Bausektor limitiert wurden ${ }^{39}$.

34 Z. B. El-Shagi El-Shagi, Neuordnung . . (Anm. 29), passim.

35 Z. B. Abdel-Malek (Anm. 19), der vom Standpunkt der unterdrückten Bauern argumentiert, und G. S. Saab (Anm. 33), der stillschweigend die enteigneten Großgrundbesitzer vertritt; cf. auch die Kritik von S. M. Gadalla (Anm. 33), pp. 86 ff.

36 Abdel-Malek (Anm. 19), pp. 118 ff.; cf. auch Khalil (Anm. 19), pp. $72 \mathrm{ff}$.

37 Abdel-Malek, op. cit., p. 119.

38 D. Warriner, Land Reform . . (Anm. 31), sowie Abdel-Malek, op. cit., p. 119.

39 Abdel-Malek, op. cit., pp. 128 f., sowie Khalil, op. cit., p. 84. 
Die Agrarreformbehörde, die sich zu einem selbständigen Ministerium entwickelt hatte, verfügte über den erwähnten „Agrarreformboden“ und hatte ein Kapital von über £E 82 Millionen. Die Beamten dieses Ministeriums, die über die Verteilung des Bodens zu bestimmen hatten, haben keine Möglichkeit privater Bereicherung versäumt. Nun hatte der Bauer, wie Abdel-Malek mit Recht hervorhebt, „nicht nur die Schikanen der Großgrundbesitzer zu ertragen, die immer eine Möglichkeit fanden, mehr Profit zu machen, sondern auch Bürokratismus und Gleichgültigkeit der Verwaltungsbeamten des Reformprogrammes, denen an nichts anderem lag, als ihren Dienst hinter sich zu bringen und darüber hinaus oftmals sich zu bereichern. Die Agrarreform war von obenher konzipiert und durchgeführt worden, um jede revolutionäre Anstrengung von seiten der Bauern zu blokkieren"40.

Nicht nur wurde das Agrarreformgesetz von korrumpierten Beamten und Großgrundbesitzern unterlaufen, sondern auch von den Bauern selbst. Der Artikel des Gesetzes, der die Parzellierung des Bodens unter 5 Feddan verbietet, erwies sich, wie schon angedeutet, als unpraktikabel, da die Erben von Kleinbauern nicht bereit waren, auf ihren Erbanteil zu verzichten, und das Gesetz zu umgehen wußten.

Sowohl außen- als auch innenpolitische Gründe haben die Regierung gezwungen, weitere gesetzliche Maßnahmen zu ergreifen. Bereits im September 1958 wurde ein Gesetz erlassen, demzufolge die Entschädigung der Großgrundbesitzer in einem Zeitraum von 40 statt wie bisher: 30 Jahren erfolgt; der Zinssatz der als Entschädigung ausgegebenen Staatsobligationen wurde von 3 auf 1,5 Prozent gesenkt, und schließlich wurden die Chargen der Enteignung und Neuverteilung von 15 auf 10 Prozent herabgesetzt ${ }^{41}$.

Als im September 1961 ein rechts gerichteter Staatsstreich in Syrien die Sezession dieser "Nordregion" der VAR herbeiführte und in Syrien die enteigneten Großgrundbesitzer und Unternehmer rehabilitierte ${ }^{42}$, wurden die Hoffnungen der ägyptischen Großgrundbesitzer auf eine ebensolche politische Veränderung in Ägypten groß; sie erwachten zu einer politischen Aktivität, so daß das Regime sich gezwungen sah, Konsequenzen aus seiner bisherigen Politik zu ziehen. In diesem Zusammenhang sind die zweite große Verstaatlichungswelle ${ }^{43}$ und die zweite Agrarreform zu sehen, die erfolgten, nachdem die erwarteten Investitionen im Industriesektor zu lange auf sich hatten warten lassen.

Das zweite Agrarreformgesetz von $1961^{44}$ reduzierte die Maximalgröße an Bodeneigentum auf 100 Feddan, einschließlich des Anteils von Angehörigen. $\mathrm{Zu} \mathrm{diesem}$ Boden rechnet nach dem neuen Gesetz auch das Brach- und Odland und nicht mehr nur kultivierter Boden wie zuvor. Die als Entschädigung hierfür ausgegebenen Staatsobligationen waren nicht mehr handelbar wie bisher. Auch konnten nach dem neuen Gesetz nurmehr kleine Grundbesitzer Land verpachten. Und das Gesetz enthielt schließlich einige Vergünstigungen für die Bauern, an die Agrarreformboden verteilt worden war.

40 Abdel-Malek, op. cit., pp. $120 \mathrm{f}$

41 Ibid., pp. 122 f

42 Hierzu Malcolm Kerr, The Arab Cold War 1958-1967, London 1968, wo Probleme der Sezession Syriens

43 Fon der VAR ausführlich behandelt werden. 44 Hierzu ausführlich D. Warriner, Land Reform... (Anm. 31), pp. 208 ff. 
Mit dem neuen Gesetz stieg der Staatsbesitz an Boden auf 1120648 Feddan an, und dem Agrarreformministerium wurde nach der Demission von Marei (cf. Anm. 33) ein Offizier vorgestellt. Die Herrschaft des Militärs begann sich nun von der politischen auf die ökonomische Ebene auszudehnen ${ }^{45}$. Im Jahr 1969 wurde das Eigentum an Boden durch ein präsidiales Dekret erneut begrenzt: auf 50 Feddan.

Die gesamtgesellschaftlichen Auswirkungen der ägyptischen Agrarreformgesetze lassen sich mit Abdel-Malek folgendermaßen charakterisieren: „Es handelte sich nicht um eine Umstellung vom Kapitalismus des kolonialen Typs auf den Sozialismus, sondern nur um einen Übergang vom veralteten kolonialen Typ des Kapitalismus, der vorwiegend agrarisch war, zu einem modernen, in erster Linie industriellen Kapitalismus, einen Ưbergang, der . . . notwendigerweise zu einer staatsdirigistischen Form führen mußte, wenn er von einem kolonisierten und damit unterentwickelten Land durchgeführt wurde ${ }^{46}{ }^{\alpha}$.

Zur ideologischen Legitimation griff das staatsdirigistische Militärregime auf eine kuriose Differenzierung des Kapitalbegriffs zurück; es betrieb eine Verherrlichung des kleinen Privateigentums gegen das große, obwohl das große Privateigentum nicht abgeschafft worden, ja der Staat sogar der größte Privateigentümer geworden war. In der "Charta der nationalen Aktion“ wird zwischen „zwei Arten des Privatbesitzes“ unterschieden, „nämlich jenem, welcher die Tore der Ausbeutung öffnet, und dem nicht ausbeutenden Privateigentum, das seine Aufgabe im Dienst der Volkswirtschaft erfüllt und dabei zugleich den Interessen der Eigentümer dient $^{\text {“47 }}$. Die Agrarreformgesetze werden in der Charta als Anwendung des „arabischen Sozialismus “ auf die Landwirtschaft bezeichnet; diese Anwendung bedeute nicht, „daß das Land nationalisiert und in Domänen, in öffentliches Eigentum umgewandelt werden muß. Aber aus der Erfahrung und gründlichem Studium der Dinge hat sich der Glaube an das Einzeleigentum am Lande in Grenzen, welche keinen Feudalismus entstehen lassen, ergeben" ${ }^{48}$. Diese ideologischen Prämissen sind auch konstitutiv für das ägyptische genossenschaftliche Experiment, das wir im folgenden behandeln werden.

\section{Agrargenossenschaftswesen und Genossenschaftsrecht in Ägypten}

Das Agrarreformgesetz von 1952 enthält mehr als den Erlaß einer Bodenreform, insofern es sich nicht nur auf eine Redistribution des Bodens beschränkt, sondern auch eine genossenschaftliche Entwicklung vorsieht und. somit in die gesamte Agrarverfassung Ägyptens zu jener Zeit eingreift ${ }^{49}$. Die aufgrund des Gesetzes geschaffenen genossenschaftlichen Gebilde nehmen in der ägyptischen Wirtschaft - besonders seit der Ausweitung des staatlichen Sektors ${ }^{50}$ - einen zentralen Platz neben dem privaten Sektor ein. Allerdings ist die Geschichte des Genossenschaftswesens in Ägypten älter als die Gesetzgebung von 1952.

45 Cf. hierzu B. Tibi, "Armee und sozialer Wandel .. (Anm. 31).

46 Abdel-Malek op. cit., p. 126; cf. ferner die Arbeit von Abdel-Malek, La Reforme agraire en Egypte (RAU): Problèmes et perspectives“, in: Dévelopment et Civilisation, Bd. 22 (1965), Juni-Heft, pp. 19-27, und dazu die Diskussion hierüber auf pp. $77 \mathrm{f}$. desselben Heftes.

47 Charta der VAR, Informationsame Kairo o.J. (deutsche Ausgabe), p. 55

48 Ibid., p. 61.

49 Zur landwirtschaftlichen Entwidklung Agyptens insgesamt ef. R. Ortner, $n$ Aspekte der landwirtschaftlichen Entwidklung Agyptens ${ }^{*}$ in: Orient, Bd. 7 (1966), H. 1, Pp. 10-15, und H. 2, pp. 50-54, und jetzt
M. R. El-Ghonemy, Economic and Institutional Organization of Egyptian Agriculture since 1952, in: P. M. Vatikiotis (ed.), Egypt since the Revolution, George Allen \& Unwinn Lrd., London 1968, pp. $66-83$.

50 Cf. den Uberblick über den ägyptischen Staatssektor in H. J. Kornrumpf, Vereinigte Arabische Republik, Leske Verlag, Opladen 1967', pp. $12 \mathrm{ff}$. 
a) Agrargenossenschaftswesen und Genossenschaftsrecht in Agypten vor 1952

Neben der bereits erwähnten Arbeit von Saab ${ }^{51}$ informierten zwei wichtige deutschsprachige Gesamtdarstellungen gründlich über das ägyptische Genossenschaftswesen: die Dissertation von Jürgen von Muralt ${ }^{52}$ sowie der Forschungsbericht von Klaus-Peter Treydte ${ }^{53}$. Aufschlußreich sind auch die auf Feldforschung basierenden Arbeiten von Wörz und El-Shagi, auf die wir im Abschnitt $\mathrm{III} / \mathrm{c}$ ) eingehen werden.

v. Muralt berichtet über die ersten Versuche, Genossenschaften in Agypten aufzubauen. Sie stammen aus der Zeit vor 1923, d. h. vor Erlaß des ersten ägyptischen Genossenschaftsgesetzes. Träger dieser Versuche waren europäisch gebildete Agypter; hervorzuheben ist Omar Lutfi. Die Pioniere des Genossenschaftswesens in Agypten hatten mit großen Schwierigkeiten zu kämpfen, denn „die konkrete Ausgestaltung des Unternehmungstyps ,Genossenschaft' kann nicht losgelöst von der herrschenden Wirtschaftsordnung betrachtet werden... Insbesondere wird die Haltung des Staates einen bedeutenden Einfluß auf die Entwicklung der Genossenschaften ausüben" ${ }^{54}$.

Die Versuche Omar Lutfis, die aus der Landaristokratie gestellte ägyptische Regierung zu einem Genossenschaftsgesetz zu bewegen, scheiterten zunächst. Dennoch wurden Genossenschaften gebildet, die nicht nur angesichts eines fehlenden Genossenschaftsgesetzes stets auf rechtliche und somit politische Schwierigkeiten stießen (cf. pp. $22 \mathrm{f}$.), sondern sich sogar gezwungen sahen, das herrschende Gesetz zu umgehen. 1923 wurde schließlich das erste ägyptische Genossenschaftsgesetz erlassen, das es sich zur Aufgabe machte, "die Entstehung landwirtschaftlicher Genossenschaften in Ägypten zu fördern und eine Grundlage für ihre Gründung und Einrichtung zu geben" (p. 30). Das Gesetz (\$2) nennt die Genossenschaftsarten wie: Bezugsgenossenschaften, Absatzgenossenschaften etc.; als Minimum für die Gründung einer Genossenschaft sind 10 Personen gefordert; als Organe sind vorgesehen: eine Generalversammlung, ein Vorstand sowie ein Kontrollausschuß. Das Gesetz war, wie v. Muralt und viele andere urteilen, nicht genossenschaftsfreundlich. Es ließ „die volle Entfaltung der Möglichkeiten der Genossenschaften als selbstbestimmende und in Selbstverwaltung stehende Förderungswirtschaften

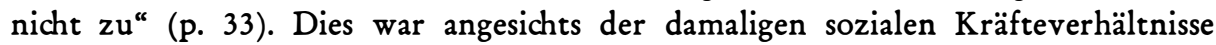
auch nicht anders zu erwarten.

Das darauffolgende zweite Genossenschaftsgesetz von 1927 ist ausführlicher und in rechtlichen Fragen genauer. Es enthält gegenüber dem ersten Gesetz viele Änderungen, hauptsächlich verwaltungsrechtlicher Art, die auch die Überwachung der Genossenschaften betreffen (cf. p. 34). Das dritte Genossenschaftsgesetz Ägyptens von 1944 enthält gegenüber dem zweiten „nur wenige und keine grundlegenden Änderungen“ (p. 38).

Unter allen drei Gesetzen konnte aber das Genossenschaftswesen, wie in der Literatur übereinstimmend berichtet wird, nicht gedeihen. Allein während der Jahre des Zweiten Weltkrieges erfuhren die Genossenschaften in Ägypten einen künst-

51 G. S. Saab, The Egyptian Agrarian Reform . . . , op. cit., pp. 51 ff., sowie M. H. A. Samie, Agrarreform und Maßnahmen : . , op. cit., pp. 83 ff.

52 Jürgen $v$. Muralt, Entwicklung und Struktur des Genossenschaftswesens in Ägypten unter besonderer Berücksichtigung der landwirtschaftlichen Genossenschaften, Michael Triltsch Verlag, Düsseldorf 1964 (zuerst als Diss., Marburg).

53 Klaus-Peter Treydte, Genossenschaften in der VAR (Ägypten). Entwicklung, Stand und Struktur des ägyptischen Genossenschaftswesens, Verlag für Literatur und Zeitgeschehen, Hannover 1971.

54 v. Muralt, op. cit., p. 14; alle folgenden Nachweise in Klammern im Text beziehen sich auf diese Quelle. 
lichen Zuwachs, besonders in den Jahren 1942/43, als die Regierung die Genossenschaften als Mittel benutzte, der durch den Krieg bedingten Preisentwicklung entgegenzuwirken. Während 1942 nur 862 Genossenschaften existierten, waren es 1944 bereits 1953 (cf. Tabelle: p. 41). Danach kam diese Aufwärtsentwicklung zum Stillstand.

Als Faktoren, die zum Versagen der Genossenschaften in Ägypten führten, nennt v. Muralt die Religion, den geringen Bildungsstand der Bauern, Korruption sowie u. a. die bestehenden Institutionen (pp. $44 \mathrm{ff}$., $48 \mathrm{ff}$.). Die Wurzel all dieser Faktoren, nämlich die spezifische Sozialstruktur Ägyptens, wird in v. Muralts Detailuntersuchung vernachlässigt. Es ist ihm aber nicht entgangen, daß vor 1952 die Genossenschaften in Ägypten sich nicht entfalten konnten, weil „die bestehenden wirtschaftlichen und sozialen Verhältnisse die Entfaltung der Selbsthilfe vieler Bevölkerungsschichten hemmten, wenn nicht sogar unmöglich machten" (p. 187). v. Muralt betont sogar, daß „die mangelhafte Genossenschaftsentwicklung in Ägypten... keinesfalls als Folge einer unzureichenden Gesetzgebung angesehen werden kann" (p. 67).

Auch Treydte zeigt, daß vor 1923 die genossenschaftliche Bewegung in Ägypten scheitern mußte, wie er es sieht, weil „eine wirklich bedeutende Genossenschaftsbewegung ohne entsprechende Gesetzgebung undenkbar ist " ${ }^{\text {55 }}$. Nach seiner Analyse der drei genannten Genossenschaftsgesetze (pp. 12 ff., 16 ff., 20 ff.) muß Treydte jedoch auch für die Zeit nach 1923 ein Scheitern des genossenschaftlichen Experiments in Kigypten konstatieren. „Die landwirtschaftlichen Genossenschaften betätigten sich hauptsächlich als Vermittler von Staatskrediten und als Verteiler von rationierten Gütern wie Saatgut, Futter, Getreide und Düngemittel. Sie vermittelten weder moderne Produktionstechniken zur Steigerung des Einkommens der Fellachen, noch bauten sie einen leistungsfähigen genossenschaftlichen Absatzapparat auf ( p. 27)." Auch Treydte unterstreicht, was schon andere Autoren feststellten ${ }^{56}$, daß die bestehenden Genossenschaften vor 1952 zugunsten der Großgrundbesitzer wirkten. „Die Genossenschaften waren nicht Organisationen der Armen, um ihre Lage zu verbessern, sondern ein Instrument des Feudalismus gegenüber den Fellachen und den Kleinbauern (p. 29)." Jedoch gelingt es Treydte nicht, diesen Tatbestand stringent abzuleiten. Er vertritt die seltame Ansicht, daß der höhere Bildungsstand der Großgrundbesitzer diese Lage verursachte, und sieht nicht, daß es sich hier nicht um Probleme der besseren Bildung handelt, sondern um typische Erscheinungsformen eines Sozialsystems, in dem das Privateigentum vorherrscht und das zudem in jeder Hinsicht unterentwickelt ist.

\section{b) Das ägyptische Genossenschaftsrecht seit 1952}

Schon das Agrarreformgesetz von 1952, das wir bereits behandelten, enthält in seinem zweiten Teil mehrere Paragraphen $(\$ 18-22)$ über die Einrichtung von Genossenschaften ${ }^{57}$ :

$\int 18$ bestimmt, daß die Bauern, an die das konfiszierte Land verteilt wird, einer Genossenschaft beitreten müssen, die jeweils in einem Dorf zu gründen ist, wobei, falls erforderlich, mehrere Genossenschaften in einem Dorf gebildet werden können. Dieser $\int 18$ läßt die Ausführungsbestimmungen für die Genossenschaftsgrün-

55 Treydte, op. cit., p. 11; alle folgenden Nachweise in Klammern im Text beziehen sich auf diese Quelle. 56 Khalil, Grundeigentum . . (Anm. 19), p. 32 ff.

57 S. Marei, op. cit. (Anm. 33), pp. 95 ff., sowie J. v. Muralt (Anm. 52), pp. 62 f. 
dung des Gesetzes Nr. 58 aus dem Jahr 1944 weiterhin gelten. $\ 19$ nennt die Aufgaben der Genossenschaften, und $\ 20$ bestimmt, daß jede gegründete Genossenschaft unter der Leitung eines "Supervisors“ (Muschrif) der Agrarreformbehörde zu stehen hat. Die Paragraphen 21 und 22 verweisen auf das Genossenschaftsgesetz von 1944 und auf künftig zu erlassende Verordnungen des Sozialministers. Jedoch wurde das Genossenschaftsgesetz von 1944 durch ein neues Gesetz im Jahr 1956 aufgehoben. v. Muralt untersucht das Gesetz von 1956 ausführlich (pp. 67 ff.) und zeigt, daß die Gründungsformalitäten und die Auflösungsvorschriften sich gegenüber dem vorherigen Gesetz, das dem deutschen Genossenschaftsrecht entsprach, kaum geändert haben. Als wesentlichen Teil des neuen Gesetzes bezeichnet v. Muralt die Bestimmung über die Regelung der Genossenschaften höherer Ordnung und Verbände. So können nach Artikel 43 zwei oder mehrere Genossenschaften zu einer Hauptgenossenschaft vereinigt werden. Artikel 45 sieht ein Minimum von 10 Genossenschaften für die Gründung eines Verbandes vor.

Bis zum Erlaß dieses Gesetzes im Jahr 1956 hatte das Nasser-Regime, sieht man von den staatlichen Agrarreform-Genossenschaften ab, sich in das allgemeine Genossenschaftswesen nicht eingemischt. Auf das Gesetz folgten 1960 eine Reihe von Präsidialverordnungen, die das Ziel hatten, das gesamte Genossenschaftswesen, das, wie v. Muralt sagt, „zu dieser Zeit. in organisatorischer Hinsicht ein verwirrendes Bild“ bot (p. 76), zu reorganisieren.

Das Gesetz Nr. 267 von 1960 sah die Gründung einer „Allgemeinen Genossenschaftsorganisation" vor, und die darauffolgenden Verordnungen wollten die Genossenschaften in die gesamte Wirtschaftsplanung des Landes einordnen; sie überwiesen die einzelnen Genossenschaften an die jeweils für sie zuständigen Ministerien zur Ưberwachung.

c) Die agrarstrukturelle Transformation Ägyptens im Schatten des neuen Genossenschaftsrechts

Nach der Ausdehnung der für die Bodenreformgenossenschaften gültigen Gesetze auf die anderen landwirtschaftlichen Genossenschaften und im Zuge der Kultivierung bisher brachliegenden Landes kristallisierten sich in Ägypten drei Genossenschaftstypen heraus: die Agrarreformgenossenschaft, die landwirtschaftlichen Genossenschaften mit alter Besitzstruktur in jenen Dörfern, die von den Bodenreformgesetzen nicht betroffen waren, und die Neusiedlungsgenossenschaften auf dem neu kultivierten Boden.

Uber die Auswirkungen der neuen Gesetze des Nasser-Regimes auf die Agrarverfassung Ägyptens, insbesondere auf die genannten Genossenschaftstypen, informieren zwei Untersuchungen, die auf eigener Feldforschung im engeren Sinne des Wortes, also nicht nur auf einer oberflächlichen Besichtigung der Genossenschaften beruhen: Johannes Wörz ${ }^{58}$ vermittelt in seiner Dissertation neben seinen empirischen Befunden einen Úberblick über die Diskussion innerhalb der Genossenschaftstheoretiker, die wir in Teil I dieser Arbeit bereits erwähnten. Auch faßt Wörz die landwirtschaftliche Entwicklung Ägyptens, insbesondere seit 1952; zusammen. Der Schwerpunkt seiner Arbeit liegt jedoch auf der empirischen

58 Johannes G. F. Wörz, Die genossenschaftlichen Produktionsförderung in Ägypten als Folgeerscheinung der Agrarreform und als neues Element genossenschaftlicher Entwidklung, Ernst Klett Verlag, Stuttgart 1967; die folgenden Seitenangaben in Klammern im Text weisen auf diese Quelle hin. 
Untersuchung, die ermitteln soll, inwiefern die Agrarstrukturen Ägyptens durch die landwirtschaftliche Neuordnung transformiert wurden. Die Untersuchung wurde in sechs Dörfern durchgeführt: in zwei Bodenreformdörfern, zwei Neusiedlerdörfern und zwei Dörfern mit alter Besitzstruktur (cf. p. 37). Zunächst untersucht Wörz die einzelnen Merkmale der ausgewählten Genossenschaften (pp. $87 \mathrm{ff}$.), ihre Agrarverfassung (pp. 96 ff.), ihre sozio-ökonomischen Merkmale (pp. $111 \mathrm{ff}$.) und ihren Aufbau. Sodann behandelt er detailliert die Auswirkungen der neuen Agrarverfassung auf sozio-organisatorischer (pp. 144 ff.), sozio-ökonomischer (pp. $162 \mathrm{ff}$.) und sozio-politischer Ebene (pp. $180 \mathrm{ff}$.).

Trotz aller Unterschiede der untersuchten Dörfer kann Wörz die allgemeine Feststellung treffen, daß ,in den Gebieten der Bodenreform... diejenigen Veränderungen, die durch die Umverteilung des Landbesitzes erfolgten, von weit größerer Bedeutung (sind) als diejenigen, die durch die genossenschaftliche Produktionsförderung bewirkt wurden" (p. 188). Er stellt sogar fest, daß die Herrschaftsstrukturen in den Dörfern mit alter Besitzstruktur sich kaum geändert haben: In den landwirtschaftlichen Genossenschaften, „wo die traditionelle Führungsgruppe aus vorrevolutionärer Zeit nahezu unverändert übernommen wurde,... konnte beobachtet werden, daß sich die betreffenden Vorstandsmitglieder bzw. der Sekretär der Genossenschaft ohne weiteres gegen den „supervisor“, in einem Falle sogar gegen dessen Vorgesetzte, behaupten konnten" (p. 190). Aber diese Dörfer haben an den ökonomischen Erfolgen der Agrarreformgenossenschaften auch teilgehabt. So wurde trotz großer Widerstände und Schwierigkeiten, von denen Wörz berichtet (pp. $72 \mathrm{ff}$.), die vereinheitlichte Fruchtfolge auch in den Dörfern mit alter Besitzstruktur im Rahmen der dort aufgebauten und schon existierenden Genossenschaften eingeführt. Hiermit waren Verbesserungen der Erträge verbunden, zumal durch die Beseitigung der unterschiedlichen Zeiträume und Rhythmen in der Entwicklung der Landfrüchte, die durch die Parzellierung bedingt waren. In diesem Zusammenhang erfolgte auch eine effektivere Bekämpfung der Schädlinge, eine bessere Bewässerung und der Einsatz von Maschinen (cf. pp. 70 f.).

Die Arbeit von El-Shagi El-Shagi ${ }^{59}$ ähnelt der von Wörz: Sie enthält zugleich eine empirische, auf eigener Feldforschung aufgebaute Studie und einen Bericht über die genossenschaftstheoretische Diskussion neben allgemeinen Informationen über die agrarwirtschaftliche Transformation in Ägypten seit 1952. Besonders folgenreich für die Ergebnisse von El-Shagis Untersuchung ist seine völlig unkritische Einstellung gegenüber dem Nasser-Regime; des öfteren läßt El-Shagi sich ungeachtet seiner empirischen Befunde in seiner als Dissertation entstandenen Arbeit von der Propaganda des Regimes blenden.

El-Shagi nennt als Grundzüge der landwirtschaftlichen Neuordnung „die Vereinheitlichung des Anbaus unter Aufrechterhaltung des. Privateigentums und die Bewirtschaftung unter genossenschaftlicher Aufsicht. Dies wurde zunächst in den aufgesiedelten Agrarreformländereien durchgeführt" (p. 35). Anschließend untersucht El-Shagi im einzelnen das System der vereinheitlichten Fruchtfolge (pp. $44 \mathrm{ff}$.) sowie die Ausführung der Pläne über die Reorganisierung der Bodennutzung, die zum Ziel hatten, „Dörfer mit traditioneller Besitzstruktur an das System in den Agrarreformgebieten anzugleichen“ (p. 45). Diese Pläne wurden 1963/64 ausge-

59 El-Shagi El-Shagi, Neuordnung und Bodennutzung in Agypten, Weltforum Verlag, München 1969; die folgenden Seitenangaben in Klammern im Text verweisen auf diese Quelle. 
führt (cf. pp. 46 ff.). Das Ergebnis war eine erhebliche Verbesserung der Flädhenerträge, wie El-Shagis Daten belegen (cf. pp. $51 \mathrm{ff}$.). Die landwirtschaftliche Neuordnung erfolgte in drei Phasen: „Reorganisation des Genossenschaftswesens und Agrarkredits (1956-1961), Einführung vereinheitlichter, kontrollierter Fruchtfolge (1961-1964), Unterstellung der örtlichen Genossenschaften unter Aufsicht und Leitung von Regierungsbeamten und vollständige Lenkung und Úberwachung des Anbaus (ab 1963) (p. 147).“

Um eine komparative Analyse durchführen zu können, konzentrierte El-Shagi seine empirische Untersuchung auf drei Dörfer, die aufgrund ihrer Agrar- und Sozialstruktur als typische ägyptische Dörfer anzusehen sind und ähnliche Verhältnisse aufweisen (cf. p. 55). Als wichtigstes Ergebnis der Untersuchung erscheint die Feststellung, daß die staatliche Bevormundung der Genossenschaften durch den "Supervisor" unpopulär ist, sowie die Feststellung, daß in den landwirtschaftlichen Genossenschaften in Dörfern mit alter Besitzstruktur kaum eine soziale Transformation stattgefunden hat, was von Wörz ebenso konstatiert wurde. Interessant ist, daß in dem Untersuchungsdorf al-Haddad bei der Befragung $63 \%$ der Bauern gegen die staatliche Uberwachung waren und daß $89 \%$ von ihnen den Ersatz des Supervisors durch einen Beauftragten des Ministeriums ablehnten (p. 119). Dennoch neigt El-Shagi dazu, die Legitimation der staatlichen Bevormundung der Bauern durch den Hinweis auf den niedrigen Stand ihres Bewußtseins und ihrer Bildung zu teilen, ohne darüber zu reflektieren, daß diese staatliche Bevormundung keine Emanzipation der Bauern von ihrer jetzigen Bewußtseinsstufe impliziert, da der Staat nichts unternimmt, um die Bevormundung in einem späteren Stadium überflüssig zu machen. Diese Frage wollen wir im folgenden abschließenden Teil unserer Arbeit wieder aufnehmen.

\section{Die Lehren des ägyptischen Agrarexperiments für die übrigen Entwicklungsländer}

Zweifellos ist die neue Agrarverfassung Agyptens trotz aller Kritik und Distanz ein Fortschritt gegenüber den Verhältnissen vor 1952. Doch läßt diese Feststellung unbefriedigt, wenn man die Problematik in ihrer Vielschichtigkeit ins Auge faßt. Die Verbesserung der Flächenerträge als einzige positive Auswirkung der landwirtschaftlichen Neuordnung erscheint uns als ein zu mageres Ergebnis. Die Befreiung der Bauern aus der Verfügung von Wucherern und Pachtherren über sie, um sie nun staatlich zu bevormunden, betrachten wir nicht als substantielle Emanzipation, abgesehen davon, daß die Wucherer und traditionellen Pachtherren nicht völlig aus den ägyptischen Dörfern verschwunden sind. Der Skandal im Dorf Kamschisch hat sogar gezeigt, daß es bisweilen zu einem Bündnis zwischen den staatlichen Behörden und den traditionellen Autoritäten auf dem Lande gegen die Bauern gekommen ist.

In den in dieser Arbeit verwerteten Untersuchungen finden wir - ausgenommen die Arbeit von El-Shagi - eine in ihrer Schärfe und Stringenz variierende Kritik. So beurteilt Abdel-Malek, der als engagierter Wissenschaftler die Interessen der Bauern in seiner Arbeit verteidigt, das ägyptische Agrarexperiment insgesamt sehr kritisch. Für ihn ist die ägyptische Agrarreform, wie bereits zitiert, ein Versuch der Überleitung des Kapitalismus kolonialen Typs in einen industriellen Kapitalismus. Die genossenschaftliche Zusammenarbeit erfolgte "auf Kreditbasis und zur Kommerzialisierung der landwirtschaftlichen Erzeugnisse... Weder der Form noch 
der Intention nach, weder als Institution noch in der Ausrichtung der Politik besteht die Tendenz zu einem Genossenschaftswesen im Landbesitz. Die Struktur und das Wesen der landwirtschaftlichen Produktion bleibt von Grund auf und in ihrem gesamten Umfang kapitalistisch." ${ }_{00}$ - Dagegen teilt El-Shagi die offizielle Version des Nasser-Regimes: „Hauptziele dieser Reform waren die Abschaffung des Feudalismus, Befreiung der Bauern von der Abhängigkeit der Großgrundbesitzer, Schaffung von möglichst vielen Kleineigentümern, Pächterschutz und Erhöhung der Landarbeiterlöhne." ${ }_{11}$ Dementsprechend beurteilt El-Shagi das Agrargenossenschaftswesen und bejaht die staatliche Bevormundung der Bauern. Treydte, der ebenso wie El-Shagi große Sympathien für das ägyptische Agrarexperiment aufbringt, nennt den Versuch „ein demokratisches Lenkungsexperiment"62, kann aber nicht umhin, kritische Punkte zu registrieren. So räumt er ein, daß der Supervisor als Vertreter des Staates die entscheidende Macht in der Genossenschaft sein könne; auch gibt Treydte zu, daß der Vorstand und die Genossenschaft bisweilen zu Zustimmungs- bzw. Ausführungsorganen degradiert sind ${ }^{63}$. An anderer Stelle meldet er ähnliche Kritik an, zieht sie aber gleich wieder zurück: „Wenn auch die Mitglieder des Genossenschaftsvorstandes und der ASU häufig noch aus der höheren Grundeigentümer- und Händlergruppe kommen, und wenn auch aufgrund besserer Ausbildung diese gehobene Schicht häufig Machtpositionen zum Beispiel in der Dorfverwaltung innehat, so ist jedoch die Situation heute nicht mehr durch die frühere Abhängigkeit gekennzeichnet. Die Führungskräfte im Genossenschaftsvorstand werden von den Bauern gewählt und können von ihnen abgewählt werden. ${ }^{64 " ~ W i e ~ d i e ~ W a h l p r a k t i k e n ~ i n ~ A ̈ g y p t e n ~ a u s s e h e n, ~ m u ̈ ß t e ~ T r e y d t e ~}$ als Ägypten-Kenner wissen. Als Beispiel für die täglichen Skandale auf diesem Gebiet sei nur auf den jüngsten Skandal im Dorf Abu-Kebir hingewiesen, wo die Bauern gegen die "demokratischen ASU-Wahlen“ auf die Barrikaden gegangen sind, nachdem ein Feudalherr mit Hilfe der lokalen staatlichen Behörden als ihr Vertreter "gewählt" werden sollte ${ }^{65}$. Treydte selbst erwähnt eine wichtige Erscheinung in den Genossenschaften, nämlich die Tendenz der Übernahme ziviler Posten durch zivil gekleidete Militärs: „Wie sehr sich der autoritäre Kurs im Staatsapparat bereits durchgesetzt hatte, war aus der zunehmenden Verwendung von Militärs für zivile Führungspositionen zu ersehen, die ihre zivile Tätigkeit auch zunehmend in Uniform ausübten. Vor 1961 beschränkte sich die Armee auf einige Schlüsselpositionen. Um 1963/64 erhielt jede Dienststelle im Land eine Sicherheitsabteilung des Abwehrdienstes zugewiesen, die nicht nur sämtliche Besucher, sondern auch die Arbeit der Dienststelle überprüft. “в6

v. Muralt geht an mehreren Stellen seiner Arbeit auf das grundsätzliche Dilemma ein, das die Geschichte der ägyptischen Genossenschaften exemplarisch für Entwicklungsländer belegt hat und das wir bereits in Teil I dieser Arbeit diskutiert haben, nämlich, „daß sich ohne eine staatliche Hilfe das Genossenschaftswesen nicht entwickeln kann, auf der anderen Seite wird durch die Staatshilfe ihr Charakter als Selbsthilfe- und Selbstverwaltungsinstitution gefährdet, denn es kann nicht

\footnotetext{
60 Abdel-Malek, op. cit., p. 126.

61 El-Shagi, op. cit., p. 31 .

62 Treydte, op. cit., p. 132

63 Ibid, p. 128.

64 Ibid., p. 127

65 Mahmud Hussein, „Intifadat Abu Kebir“ (Der Aufstand von Abu Kebir), in: Al-Hurria (Beirut), Bd. 12 (1971), H. 582, p. 7. Von Hussein liegt in französischer. Sprache folgende Studie vor: La lutte de classes en Egypte de 1945 a 1968, Edition F. Maspero, Paris 1970.

66 Treydte, op. cit., p. 131; cf. auch B. Tibi, "Armee und sozialer Wandel . . .“ (Anm. 31).
} 
erwartet werden, daß der Staat riesige Summen zur Entwicklung der Genossenschaften zur Verfügung stellt, ohne eine Kontrolle über deren Verwendung zu haben. ${ }^{67 " ~ D e r ~ F a l l ~ A ̈ g y p t e n ~ z e i g t ~ s e h r ~ d e u t l i c h, ~ d a ß ~ d i e ~ G e n o s s e n s c h a f t e n ~ k e i n e r l e i ~}$ Eigenständigkeit haben, obwohl das ägyptische Genossenschaftsrecht die Selbstverwaltung der Genossenschaften anerkennt. Die Genossenschaften sind nur Instrumente der staatlichen Politik ${ }^{68}$. In diesem Zusammenhang weist v. Muralt auf die patriarchalische Einstellung der Gesetzgeber und der staatlichen Behörden gegenüber den Bauern hin, die nicht auf ein Interesse an der Emanzipation der Bauern hindeutet ${ }^{69}$. So findet sich v. Muralt, der Sympathie für das ägyptische Agrarexperiment hegt und es auch ausführlich würdigt, schließlich gezwungen, eine grundsätzliche Kritik vorzutragen: „Weniger die Tatsache selbst, daß die Regierung Ägyptens das Genossenschaftswesen weitgehend kontrolliert, ist abzulehnen, als vielmehr die Form, in der dies geschieht. Die allzu häufige Änderung der Genossenschaftspolitik und die Bevormundung an falscher Stelle zerstören oft gute Ansätze erfolgreicher Arbeit. Dies sind wesentliche Gründe, warum es noch zu keiner Konsolidierung der Genossenschaftsbewegung gekommen ist. Trotz aller Anstrengungen konnten die Genossenschaften die Lage der Bauern noch nicht grundlegend verbessern."

Auch Wörz formuliert Kritik an der Intervention des Staates in der Person des Supervisors: Wörz konnte beobachten, „in welch hohem Maße die täglichen Anforderungen und die laufenden Entscheidungen auf den ,supervisor ${ }^{6}$ zukommen und wie sehr diese "supervisors" zu den zentralen und bestimmenden Faktoren in den Genossenschaften gehören. Dies, obwohl der „supervisor" ganz zweifellos kein genossenschaftliches, sondern ein staatliches Organ ist" 71 .

Es ist erwähnenswert, daß die zitierten Autoren, obwohl stark beeinflußt von der deutschen Genossenschaftstheorie, doch die in dieser Theorie übliche dogmatische Position, nach der Genossenschaften nur als freiwillige Selbsthilfeorganisationen definiert und alle anderen Einrichtungen als Pseudogenossenschaften verurteilt werden, zum Teil überwunden haben. Ihre Kritik richtet sich demnach nicht gegen den Tatbestand der staatlichen Intervention und der unfreiwilligen Mitgliedschaft in den Genossenschaften als vielmehr gegen die autoritäre Bevormundung der Bauern durch den Staat und seine zivil gekleideten Militärs, d. h. sie wendet sich gegen die Herrschaftsposition der Staatsträger gegenüber den Bauern, die durch die agrarstrukturelle Transformation unter dem Nasser-Regime keineswegs befreit wurden.

Angesichts der kritischen Stimmen auch von Autoren, die das ägyptische Agrarexperiment grundsätzlich anerkennen, erscheint es legitim zu fragen, ob eine agrarstrukturelle Transformation, die sich darin erschöpft, die Flächenerträge zu erhöhen, und, statt die Emanzipation der Bauern herbeizuführen, sich damit begnügt, alte Abhängigkeiten durch neue zu ersetzen, wirklich als Modellfall für andere Länder dargestellt und zur Nachahmung empfohlen werden kann. Die vorliegenden Materialien lassen den Schluß zu, daß die ägyptischen Bauern von der heutigen militär-bürokratischen Herrschaft ebenso unterdrückt werden wie ehedem unter der Monarchie.

$67 \mathrm{~J}$. v. Muralt, op. cit., pp. $90 \mathrm{f}$.

68 Ibid, p. 94.

69 Ibid., pp. 114 f.

70 Ibid., pp. $188 \mathrm{f}$.

71 J. F. F. Wörz, op. cit., p. 176. 
Weder das Agrarreformgesetz von 1952 und seine Zusätze noch die genossenschaftlichen Verordnungen und die Realitäten, die sie schafften, brachten der ägyptischen Landbevölkerung Freiheit und eine substantielle Verbesserung ihrer Lebensverhältnisse. Der Ausbau des Staates zu einer Herrschaftsmaschine der Armee und die Ausdehnung der Kompetenzen der Militärs auf alle Lebensbereiche, auch auf die genossenschaftlichen Führungsorgane, sind kein Indiz dafür, daß die landwirtschaftliche Neuordnung in Agypten eine fortschrittliche Transformation darstellt. So kann das viel zitierte ägyptische Agrarexperiment nicht als Vorbild einer agrarstrukturellen Transformation für die übrigen Entwicklungsländer gelten. 
attempts to put pressure on the Western powers to alleviate Africa's burden of apartheid and colonialism, so far without success. The British agreement with Rhodesia on 24 November 1971 illustrates the point.

\section{The Preamble of the New Draft Constitution of the People's Republic of China}

\section{By Dieter Heinzig}

In 1970, the Taiwan Intelligence Bureau managed to procure from the mainland the text of a new draft constitution of the People's Republic of China, which had been, in principle, adopted by the Second Plenary Session of the CCP Central Committee, but up to this day has not been publicly mentioned in Communist China. This first text did not include a preamble, however, and turned out to be incomplete insofar. In the meantime, the Taipei journal "Chung-kung yen-chiu" (Studies on Chinese Communism) published two versions of this missing preamble, which are nearly identical. The present article supplies a German translation of the preamble together with an introductory comment.

Compared with the 1954 constitution, the new preamble is marked generally by a more revolutionary and militant keynote thus reflecting the spirit of the Cultural Revolution. Some of its passages are verbatim quotations from Mao Tse-tung or Lin Piao, others are taken from the Chinese polemics against the Soviet Union. The inclusion of the term "social imperialism" must be regarded as a constitutional confirmation of anti-sovietism. The use of the wording "continuous" instead of "permanent revolution", when describing the domestic scene, possibly could evidence a victory won by a Lin Piao faction over a Ch'en Po-ta faction in the fall of 1970. After Lin Piao has disappeared from the political stage, the new draft constitution will have to be changed before being adopted by the forthcoming IVth National People's Congress meanwhile postponed to a still unknown date. For Lin Piao is mentioned both in the draft preamble and in the draft constitution itself in his capacity as Vice-Chairman of the Party's Central Committee and as Mao Tse-tung's successor.

\section{Agrarian Reform and Cooperatives in a Developing Country: The Case of Egypt}

\section{By Bassam Tibi}

The above study begins with an analysis of the terms agrarian reform and agrarian cooperative. The analysis is oriented on the socialeconomic situation of underdeveloped countries. It is shown that the agrarian question is of central importance because these countries are predominately agrarian regions. The vague assertation of agrarian reform to be either technical modernization, or simple redistribution of the land is critizised. An agrarian reform can only be effective when it contains both these aspects. The development of underdeveloped agrarian regions necessitates an agrarstructural transformation, but such transformation can only take place when the dominating ruling structures that could prevent it have been overcome.

These historically oriented thesises can be verified in the case of Egypt. The land laws and the agrarian question connected to them must be analyzed in order to 
reconstruct the background for the First Egyptian Agrarian Reform. The agrarian reform laws of 1952 and the Second Egyptian Agrarian Reform of 1961 are treated in a detailed study. The second part of the empirical analysis has the movement of the agrarian cooperative in Egypt as its object, that is the development of the Egyptian cooperative laws, their social origins and the effect of their application before as well as after 1952 are decribed. Several recently held field studies in the Egyptian agrarian cooperatives allow an interpretation of the results of the Egyptian agrarian experiment in the last part of the study. The author maintains that the agrarstructural change under the Nasser regime led to the modification of certain ruling structures and brought about a modernization of production and an increase of the average yield. Nevertheless a comprehensive transformation did not take place. The rule of the land aristocracy over the peasants has been substituted by the rule of the bureaucracy and its state apparatus. The social structures of rural Egypt have not been substantially changed by the agrarian reform. Most importantly the basic principle of the cooperative idea, egalitarianism, can not be found in any of the agrarian cooperatives. 